\title{
A low-complexity user selection scheme in a multicell MIMO environment
}

\author{
Doohee Kim¹, Oh-Soon Shin² and Kwang Bok Lee ${ }^{1 *}$
}

\begin{abstract}
An efficient user selection scheme for the downlink of multiuser MIMO systems is proposed in a multicell environment. In a multicell environment, the intercell interference is one of the most influential factors limiting the performance. Thus, a user selection scheme that considers intercell interference is essential to increase the sum rate. The proposed scheme is based on an interference-aware precoding. It sequentially selects users such that the sum rate is maximized. In particular, we develop a simple incremental metric for the sum rate. The use of the derived metric enables a significant reduction in the computational complexity of the user selection process, as compared to the optimal exhaustive search. Numerical results show that the proposed scheme provides near-optimal performance with substantially reduced complexity.
\end{abstract}

\section{Introduction}

Intercell interference is one of the most challenging issues limiting the performance of cellular systems, especially when the spectrum is highly reused across cells. In multiple-input multiple-output (MIMO) systems, in particular, it has been reported that in a multicell environment, the performance of spatial multiplexing is significantly degraded due to intercell interference [1]. Recently, there have been several works on multicell MIMO that attempt to mitigate the effect of intercell interference [1-5]. Most of the works have focused on precoding or beamforming strategies. For instance, centralized precoding schemes were proposed for a case in which the channel state information (CSI) is available at the transmitter [2] and for the case when it is not available [4]. In [5], a distributed precoding scheme was proposed by introducing a new precoding metric known as the signal-to-generatedinterference-plus-noise ratio (SGINR).

Precoding schemes rely on the condition that the set of users to be served is given. In practice, an appropriate choice of users may have a substantial impact on the overall system performance in multiuser MIMO scenarios [6-8]. A general framework for user selection was developed in [6] based on convex utility functions. In [7],

\footnotetext{
*Correspondence: klee@snu.ac.kr

1 School of Electrical and Computer Engineering and INMC, Seoul National University, 151-742 Seoul, South Korea

Full list of author information is available at the end of the article
}

successive user selection schemes were proposed along with the optimization of transmit beamforming vectors. In [8], various low-complexity user selection schemes were proposed. However, these schemes may suffer from severe performance degradation in multicell MIMO systems, as they do not take the intercell interference into account. This motivates us to investigate a user selection scheme applicable to multicell MIMO systems.

In this article, we propose a user selection scheme that works jointly with the SGINR-based precoding scheme in [5] for a downlink multicell MIMO system. The SGINR precoding is also known as the SLNR (signal-toleakage-and-noise ratio) precoding [3], which was discussed in 3GPP LTE-Advanced [9]. The proposed scheme is designed to select users in a successive manner such that the sum rate is maximized. We derive a simple incremental metric, which enables the system to determine whether adding a particular user would increase the sum rate or not. Owing to the derived metric, the proposed scheme requires considerably reduced computational complexity as compared to the optimal exhaustive search over all possible combinations of users. Numerical results will be presented to validate the performance of the proposed user selection scheme under various environments.

The rest of this article is organized as follows. Section 2 describes the system model. In Section 3, we develop an efficient user selection algorithm by deriving a simple increment metric for the sum rate. Numerical results

\section{Springer}

(c) 2013 Kim et al.: licensee Springer. This is an Open Access article distributed under the terms of the Creative Commons Attribution License (http://creativecommons.org/licenses/by/2.0), which permits unrestricted use, distribution, and reproduction in any medium, provided the original work is properly cited. 
are presented in Section 4, and conclusions are drawn in Section 5.

\section{System model}

We consider the downlink of a cellular system comprised of $L$ cells or sectors and $K$ users per cell or sector. Each base station (BS) and each mobile station (MS) are assumed to be equipped with $N_{\mathrm{t}}$ transmit antennas and $N_{\mathrm{r}}$ receive antennas, respectively. The $L$ cells or sectors are grouped into a cluster, as depicted in Figure 1 for the case of $L=3$. Each BS is assumed to serve only the users in its own cell using the interference-aware precoding scheme in [5]. The received signal vector $\mathbf{y}_{i}^{(k)}$ at the $k$ th MS of the $i$ th cell can be expressed as

$$
\begin{aligned}
\mathbf{y}_{i}^{(k)}= & \sqrt{\rho_{i}^{(k)}} \mathbf{H}_{i, i}^{(k)} \mathbf{W}_{i}^{(k)} \mathbf{x}_{i}^{(k)}+\sum_{j=1, j \neq k}^{K} \sqrt{\rho_{i}^{(k)}} \mathbf{H}_{i, i}^{(k)} \mathbf{W}_{i}^{(j)} \mathbf{x}_{i}^{(j)} \\
& +\sum_{m=1, m \neq i}^{L} \sum_{i=1}^{K} \sqrt{\eta_{i, m}^{(k)}} \mathbf{H}_{i, m}^{(k)} \mathbf{W}_{m}^{(j)} \mathbf{x}_{m}^{(j)}+\mathbf{n}_{i}^{(k)},
\end{aligned}
$$

where $\mathbf{x}_{i}^{(k)}$ denotes the transmit symbol destined for the $k$ th MS in the $i$ th cell, $\mathbf{W}_{i}^{(k)}$ is the corresponding precoding matrix, $\mathbf{H}_{i, m}^{(k)}$ is an $N_{\mathrm{r}} \times N_{\mathrm{t}}$ channel matrix between the BS in the $m$ th cell and the $k$ th MS in the $i$ th cell. The entries of the channel matrix $\mathbf{H}_{i, m}^{(k)}$ are assumed to follow independent and identically distributed (i.i.d) complex Gaussian distribution with zero mean and unit variance. $\mathbf{n}_{i}^{(k)}$ denotes the additive white Gaussian noise (AWGN), $\rho_{i}^{(k)}$ denotes the signal-to-noise ratio (SNR) of the $k$ th MS in the $i$ th cell, and $\eta_{i, m}^{(k)}$ denotes the interference-to-noise ratio (INR) for the interference that the BS in the $m$ th cell causes at the $k$ th MS in the $i$ th cell. The transmit symbols and noise are assumed to be normalized to have unit power. We assume that every BS has the same transmit power $P$ and that each BS allocates equal transmit power to the selected users in the corresponding cell; when $M$ users are selected from a BS, each user is allocated to the power of $P / M$. The first term in (1) corresponds to desired signal, and the second and third terms represent the intracell interference and intercell interference, respectively, within a cluster. Note that both the intracell interference and intercell interference constitute the intracluster interference, and the intercluster interference is not considered.

The cell coordination model considered in this paper falls into the category of coordinated scheduling/coordinated beamforming $(\mathrm{CS} / \mathrm{CB})$ in the context of LTEAdvanced. In a CS/CB, BSs need to share only the CSI through a coordinator, and each BS serves users in its own cell [10]. We assume that the precoding matrix $\mathbf{W}_{i}^{(k)}$ for each MS in (1) is formed based on the SGINR criterion [5]. It was shown that the SGINR-based beamforming improves the sum rate in a multicell environment. Specifically, the SGINR covariance matrix $\mathbf{K}_{\mathbf{G I}}{ }_{i}{ }^{(k)}$ at the $k$ th MS in the $i$ th cell is defined as

$$
\mathbf{K}_{\mathbf{G I}}{ }^{(k)}=\rho_{i}^{(k)}\left(\mathbf{I}_{N_{t}}+\mathbf{G}_{i}^{(k)^{H}} \mathbf{G}_{i}^{(k)}\right)^{-1}\left(\mathbf{H}_{i, i}^{(k)}{ }^{H} \mathbf{H}_{i, i}^{(k)}\right),
$$

where

$$
\mathbf{G}_{i}^{(k)} \equiv\left[\left(\mathbf{H}_{\mathbf{G}_{1, i}}^{(k)}\right)^{T} \cdots\left(\mathbf{H}_{\mathbf{G}_{i, i}}^{(k)}\right)^{T} \cdots\left(\mathbf{H}_{\mathbf{G}_{L, i}}^{(k)}\right)^{T}\right]^{T}
$$

denotes a composite matrix containing both the intercell interference channels and the intracell interference channel that the $k$ th MS in the $i$ th cell may cause to other users, $(\cdot)^{H}$ denotes the conjugate transpose, and $(\cdot)^{T}$ denotes the transpose of a matrix. Each submatrix of $\mathbf{G}_{i}^{(k)}$ is defined as

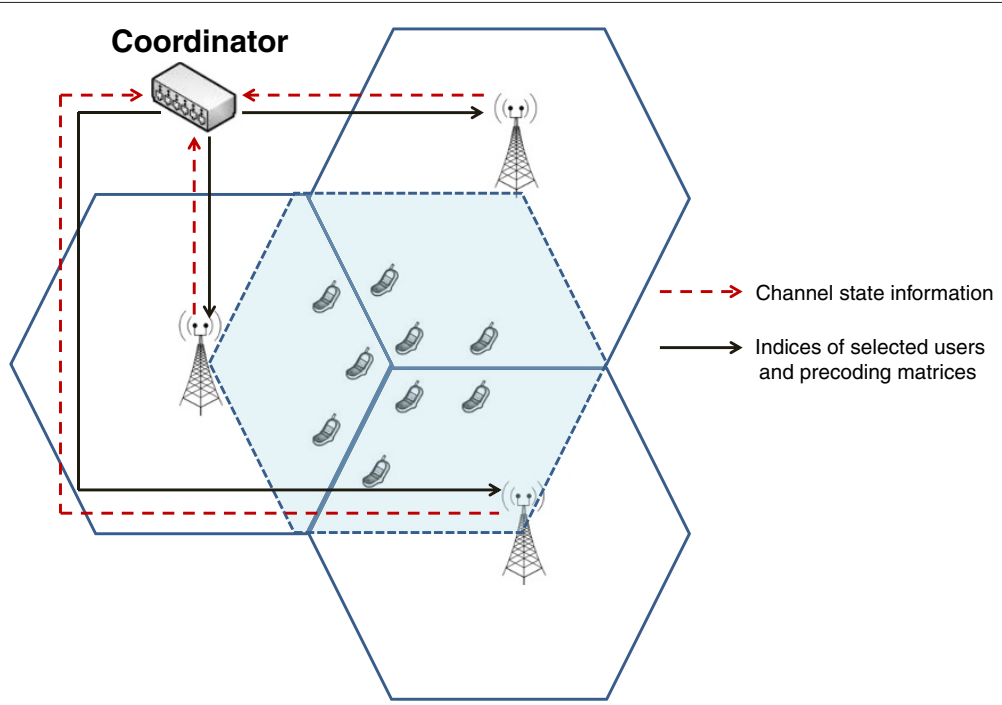

Figure 1 A cluster of three sectors for a multicell system. 


$$
\mathbf{H}_{\mathbf{G}_{j, i}^{(k)}}=\left\{\begin{array}{l}
{\left[\sqrt{\rho_{i}^{(1)}} \mathbf{H}_{i, i}^{(1)} \cdots \sqrt{\rho_{i}^{(k-1)}} \mathbf{H}_{i, i}^{(k-1)} \sqrt{\rho_{i}^{(k+1)}} \mathbf{H}_{i, i}^{(k+1)} \cdots \sqrt{\rho_{i}^{(K)}} \mathbf{H}_{i, i}^{(K)}\right], \quad i=j} \\
{\left[\sqrt{\eta_{j, i}^{(1)}} \mathbf{H}_{j, i}^{(1)} \cdots \sqrt{\eta_{j, i}^{(K)}} \mathbf{H}_{j, i}^{(K)}\right], \quad i \neq j .}
\end{array}\right.
$$

To construct a beamforming matrix $\mathbf{W}_{i}^{(k)}$, we express $\mathbf{K}_{\mathbf{G I}}{ }_{i}{ }^{(k)}$ in (2) using the eigenvalue decomposition as

$$
\mathbf{K}_{\mathbf{G I}}{ }_{i}^{(k)}=\mathbf{V}_{\mathbf{G I}}{ }_{i}^{(k)} \mathbf{D}_{\mathbf{G I}}{ }_{i}^{(k)} \mathbf{V}_{\mathbf{G I}}{ }_{i}^{(k)}{ }^{H}
$$

where $\mathbf{D}_{\mathbf{G I}}{ }_{i}^{(k)}$ and $\mathbf{V}_{\mathbf{G I}}{ }_{i}^{(k)}$ denote the diagonal matrix composed of eigenvalues and unit-norm eigenvector matrix of $\mathbf{K}_{\mathbf{G I}}{ }_{i}^{(k)}$, respectively. Then, $\mathbf{V}_{\mathbf{G I}}{ }_{i}^{(k)}$ corresponds to a beamforming matrix $\mathbf{W}_{i}^{(k)}=\mathbf{V}_{\mathbf{G I}}{ }^{(k)}{ }^{(k)}$ [5]. Thus, the precoding and user selection require centralized processing, i.e., CSI sharing among cells. As illustrated in Figure 1, each BS in a cluster collects information on both the desired channel and interference channels for each MS. For the $k$ th MS in the $i$ th cell, for example, $\mathbf{H}_{i, i}^{(K)}$ corresponds to the desired channel matrix, and $\mathbf{H}_{\mathbf{G}_{j, i}}{ }^{(k)}, j \neq i$ corresponds to interference channel matrices. It is assumed that the MS perfectly estimates both the desired and interference channels and feeds them back to the BS without error. The BS transports the CSI to a cluster coordinator, which then performs the beamforming and user selection and notifies the results back to the associated BSs.

Assuming that each MS employs the maximum likelihood (ML) detection, the achievable rate $R_{i}^{(k)}$ of the $k$ th MS in the $i$ th cell can be computed as

$$
R_{i}^{(k)}=\log _{2} \operatorname{det}\left(\mathbf{I}_{N_{r}}+\Lambda_{i}^{(k)}\right)
$$

where $\Lambda_{i}^{(k)}$ denotes a matrix associated with the received signal-to-interference-plus-noise ratio (SINR) of the $k$ th MS in the $i$ th cell [2]. It is given as

$$
\Lambda_{i}^{(k)}=\rho_{i}^{(k)}\left(\mathbf{H}_{i, i}^{(k)} \mathbf{W}_{i}^{(k)}\right)\left(\Gamma_{i}^{(k)}\right)^{-1}\left(\mathbf{H}_{i, i}^{(k)} \mathbf{W}_{i}^{(k)}\right)^{H}
$$

where $\Gamma_{i}^{(k)}$ represents the noise plus interference power

$$
\begin{aligned}
\Gamma_{i}^{(k)}= & \mathbf{I}_{N_{r}}+\sum_{j=1, j \neq k}^{K} \rho_{i}^{(j)}\left(\mathbf{H}_{i, i}^{(j)} \mathbf{W}_{i}^{(j)}\right)\left(\mathbf{H}_{i, i}^{(j)} \mathbf{W}_{i}^{(j)}\right)^{H} \\
& +\sum_{m=1, m \neq i}^{L} \sum_{j=1}^{K} \eta_{i, m}^{(j)}\left(\mathbf{H}_{i, m}^{(j)} \mathbf{W}_{m}^{(j)}\right)\left(\mathbf{H}_{i, m}^{(j)} \mathbf{W}_{m}^{(j)}\right)^{H}
\end{aligned}
$$

On the right hand side of (8), the first term is due to the AWGN, and the second and third terms represent the intracell interference and intercell interference, respectively. Using (6), the corresponding sum rate $\mathcal{R}_{\text {sum }}$ for all MS's in the cluster of $L$ cells is given by

$$
\mathcal{R}_{\text {sum }}=\sum_{i=1}^{L} \sum_{k=1}^{K} R_{i}^{(k)}
$$

\section{Proposed user selection scheme}

In this section, we propose a user selection scheme that works in a multicell environment. Because the achievable rate in (6) is affected by the intercell interference as well as by the intracell interference, it may not be optimal in terms of the sum rate that each BS serves all $K$ users at the same time. Therefore, the proper selection of simultaneously served users will be important to optimize the overall performance. The greedy user selection algorithm in [11] is known to provide near-optimal sum rate performance in a single-cell scenario. However, the greedy algorithm does not consider the intercell interference, which may lead to inevitable performance degradation in a multicell environment. In [2], it was shown that the optimal solution approaches a single stream transmission scheme as the intercell interference becomes strong.

We propose a user selection algorithm that maximizes the sum rate in (9) in a multicell environment. Let $U_{i} \equiv$ $\{(i, 1),(i, 2), \cdots,(i, K)\}$ represent the set of all users in the $i$ th cell, and let $\mathcal{S}_{i}^{*}$ be the set of selected users in the $i$ th cell. The user selection problem can then be formulated as

$$
\mathcal{S}^{*}=\underset{\mathcal{S}_{i} \subset U_{i}, i=1,2, \cdots, L}{\arg \max } \sum_{i=1}^{L} \sum_{k \in \mathcal{S}_{i}} R_{i}^{(k)}
$$

where $\mathcal{S}^{*} \equiv \mathcal{S}_{1}^{*} \cup \mathcal{S}_{2}^{*} \cup \cdots \cup \mathcal{S}_{L}^{*}$ denotes the resulting set of all selected users. The solution of (10) will require an exhaustive search over all possible combinations of users, which may cause high computational complexity. As a more practical solution to (10), we propose a suboptimal successive user selection scheme based on the SGINR precoding. We explain the details of the user selection criterion and the selection algorithm in the following subsections. 


\subsection{User selection criterion}

Given that the proposed user selection scheme successively adds users to the set of its served users one-by-one, we need to develop a criterion to determine whether to add a user or not at each step. Suppose that $(n-1)$ $(n \geq 2)$ users are already selected and they are represented by a set of the user indices, $\mathcal{S}^{*}(n-1) \equiv$ $\left\{\left(i_{1}, k_{1}\right),\left(i_{2}, k_{2}\right), \cdots,\left(i_{n-1}, k_{n-1}\right)\right\}$, where $i_{j}$ and $k_{j}$ represent the BS index and MS index of the $j$ th selected user, respectively. Let $\Delta R(n)$ be the increment in the sum rate when user $\left(i_{n}, k_{n}\right)$ is added to the set $\mathcal{S}^{*}(n-1)$ to form $\mathcal{S}^{*}(n)=\mathcal{S}^{*}(n-1) \cup\left\{\left(i_{n}, k_{n}\right)\right\}$. Then, $\Delta R(n)$ can be expressed as

$$
\begin{aligned}
\Delta R(n)= & \sum_{(i, k) \in \mathcal{S}^{*}(n)} \log _{2} \operatorname{det}\left(\mathbf{I}_{N_{r}}+\Lambda_{i}^{(k)}(n)\right) \\
& -\sum_{(i, k) \in \mathcal{S}^{*}(n-1)} \log _{2} \operatorname{det}\left(\mathbf{I}_{N_{r}}+\Lambda_{i}^{(k)}(n-1)\right),
\end{aligned}
$$

where $\sum_{(i, k) \in \mathcal{S}^{*}(n)}$ indicates that the sum is taken over $n(i, k)$ pairs associated with the user indices in $\mathcal{S}^{*}(n)$ when the $n$ users in $\mathcal{S}^{*}(n)$ are simultaneously served. We approximate $\Delta R(n)$ in (11) as

$$
\begin{aligned}
\Delta R(n) \approx & \sum_{(i, k) \in \mathcal{S}^{*}(n)} \log _{2} \operatorname{det}\left(\Lambda_{i}^{(k)}(n)\right) \\
& -\sum_{(i, k) \in \mathcal{S}^{*}(n-1)} \log _{2} \operatorname{det}\left(\Lambda_{i}^{(k)}(n-1)\right),
\end{aligned}
$$

which follows from the assumption that the selected users have high SINR ${ }^{\mathrm{a}}[5]$.

Then $\Delta R(n)$ in (12) can be rewritten and upper bounded as

$$
\begin{aligned}
\Delta R(n) & \approx \log _{2}\left[\frac{\left(\frac{S_{1}(n)}{I_{1}(n)}\right) \cdots\left(\frac{S_{n}(n)}{I_{n}(n)}\right)}{\left(\frac{S_{1}(n-1)}{I_{1}(n-1)}\right) \cdots\left(\frac{S_{n-1}(n-1)}{I_{n-1}(n-1)}\right)}\right] \\
& \leq \log _{2}\left[\left(\prod_{\ell=1}^{n-1} \frac{I_{\ell}(n-1)}{I_{\ell}(n)}\right) \frac{S_{n}(n)}{I_{n}(n)}\right],
\end{aligned}
$$

where $S_{\ell}(n)$ and $I_{\ell}(n)$, respectively, represent the received signal component and the noise plus interference component for the $\ell$ th selected user, when the $n$ users in $\mathcal{S}^{*}(n)$ are served simultaneously. These can be expressed as

$$
\begin{aligned}
S_{\ell}(n) & =\operatorname{det}\left(\rho_{i_{\ell}}^{\left(k_{\ell}\right)}\left(\mathbf{H}_{i_{\ell}, i_{\ell}}^{\left(k_{\ell}\right)} \mathbf{W}_{i_{\ell}}^{\left(k_{\ell}\right)}\right)\left(\mathbf{H}_{i_{\ell}, i_{\ell}}^{\left(k_{\ell}\right)} \mathbf{W}_{i_{\ell}}^{\left(k_{\ell}\right)}\right)^{H}\right), \\
I_{\ell}(n) & =\operatorname{det}\left(\mathbf{R}_{\mathbf{I} \ell}(n)\right),
\end{aligned}
$$

where $\mathbf{R}_{\mathbf{I} \ell}(n)$ denotes the noise plus received interference matrix, when the $n$ users in $\mathcal{S}^{*}(n)$ are served. The notation $i_{\ell}$ indicates the $\ell$ selected user associated to the BS in the $i$ cell. The matrix $\mathbf{R}_{\mathbf{I} \ell}(n)$ can be expressed as

$$
\begin{aligned}
\mathbf{R}_{\mathbf{I} \ell}(n)= & \mathbf{I}_{N_{r}}+\sum_{(i, k), i=i_{\ell}} \rho_{i_{\ell}}^{\left(k_{\ell}\right)}\left(\mathbf{H}_{i_{\ell}, i_{\ell}}^{\left(k_{\ell}\right)} \mathbf{W}_{i}^{(k)}\right)\left(\mathbf{H}_{i_{\ell}, i_{\ell}}^{\left(k_{\ell}\right)} \mathbf{W}_{i}^{(k)}\right)^{H} \\
& +\sum_{(i, k), i \neq i_{\ell}} \eta_{i_{\ell}, i}^{\left(k_{\ell}\right)}\left(\mathbf{H}_{i_{\ell}, i}^{\left(k_{\ell}\right)} \mathbf{W}_{i}^{(k)}\right)\left(\mathbf{H}_{i_{\ell}, i}^{\left(k_{\ell}\right)} \mathbf{W}_{i}^{(k)}\right)^{H}
\end{aligned}
$$

and $\mathbf{W}_{i}^{(k)}$ denotes the SGINR precoding matrix when $n$ users in $\mathcal{S}^{*}(n)$ are simultaneously served. Note that the inequality in (13) is due to $\prod_{\ell=1}^{n-1} S_{\ell}(n) \leq \prod_{\ell=1}^{n-1} S_{\ell}(n-1)$, which follows from the term-by-term inequalities $S_{\ell}(n) \leq$ $S_{\ell}(n-1), \ell=1,2, \cdots, n-1$. The term-by-term inequalities are valid due to the following reasons. First, when the $n$th user is added up, $n-1$ users that are already selected should be allocated to transmit power equal to or less than the value for the case when only $n-1$ users are selected, owing to the assumption of transmit power in Section 2. Moreover, when the $n$th user is added, it generates additional interference to the other $n-1$ users, and thus will decrease the received signal power of each MS.

We further assume $\sum_{\ell=1}^{n-1} I_{\ell}(n-1) \leq(n-2) I_{G}(n)$ and $\sum_{\ell=1}^{n} I_{\ell}(n) \leq n I_{G}(n)$, where $I_{G}(n)$ denotes the noise plus generated interference component of the $n$th selected user

$$
\begin{aligned}
I_{G}(n)= & \operatorname{det}\left(\mathbf{G}_{\mathbf{I}_{n}}(n)\right) \\
\mathbf{G}_{\mathbf{I}_{n}}(n)= & \mathbf{I}_{N_{r}}+\sum_{(i, k), i=i_{n}} \rho_{i}^{(k)}\left(\mathbf{H}_{i, i}^{(k)} \mathbf{W}_{i_{n}}^{\left(k_{n}\right)}\right)\left(\mathbf{H}_{i, i}^{(k)} \mathbf{W}_{i_{n}}^{\left(k_{n}\right)}\right)^{H} \\
& +\sum_{(i, k), i \neq i_{n}} \eta_{i, i_{j}}^{(k)}\left(\mathbf{H}_{i, i_{n}}^{(k)} \mathbf{W}_{i_{n}}^{\left(k_{n}\right)}\right)\left(\mathbf{H}_{i, i_{n}}^{(k)} \mathbf{W}_{i_{n}}^{\left(k_{n}\right)}\right)^{H} .
\end{aligned}
$$

The assumptions are reasonable in that the generated interference power of the last selected user will be greater than those of the previously selected users, because all users have similar received signal power and the user selection is performed sequentially based on the SGINR. Figure 2 verifies that the $\sum_{\ell=1}^{n-1} I_{\ell}(n-1)$ to $(n-2) I_{G}(n)$ ratio and the $\sum_{\ell=1}^{n} I_{\ell}(n)$ to $n I_{G}(n)$ ratio are less than unity in the typical SNR range. Using the inequality of the arithmetic and geometric means with the two assumptions, we can derive the following two inequalities:

$$
\begin{aligned}
\prod_{\ell=1}^{n-1} I_{\ell}(n-1) & \leq\left(\frac{1}{n-1} \sum_{\ell=1}^{n-1}\left(I_{\ell}(n-1)\right)\right)^{n-1} \\
& \leq\left(\frac{n-2}{n-1} I_{G}(n)\right)^{n-1}, \\
\prod_{\ell=1}^{n} I_{\ell}(n) & \leq\left(\frac{1}{n} \sum_{\ell=1}^{n}\left(I_{\ell}(n)\right)\right)^{n} \leq\left(I_{G}(n)\right)^{n} .
\end{aligned}
$$




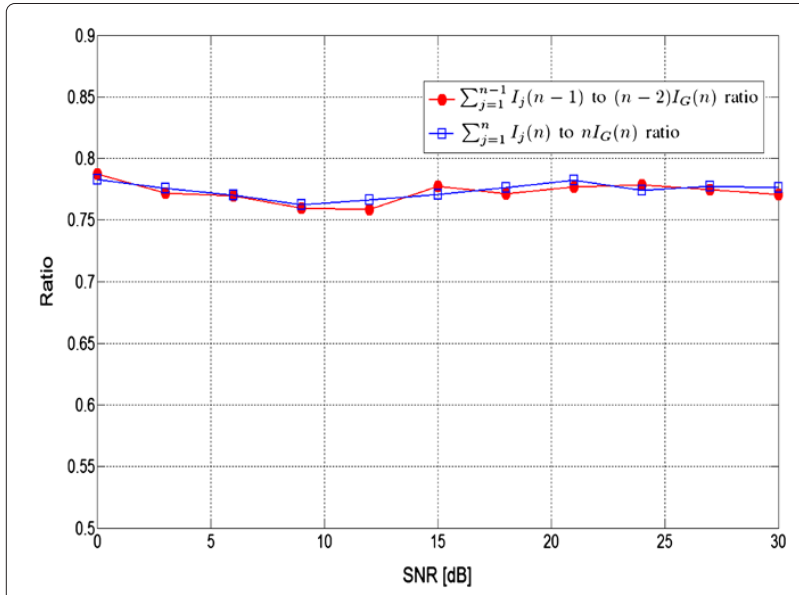

Figure $2 \sum_{j=1}^{n-1} I_{j}(n-1)$ to $(n-2) I_{G}(n)$ ratio and $\sum_{j=1}^{n} I_{j}(n)$ to $n I_{G}(n)$ ratio vs. SNR, when $K=10, N_{t}=4$ and $N_{r}=2$.

Using the upper bounds in (13) and (17), we approximate $\Delta R(n)$ as

$$
\Delta R(n) \approx \log _{2}\left[\frac{\left(\frac{n-2}{n-1} I_{G}(n)\right)^{n-1} S_{n}(n)}{\left(I_{G}(n)\right)^{n}}\right], \quad n \geq 2
$$

Accordingly, we define the metric $\Delta r(n)$ as

$$
\Delta r(n) \equiv 2^{\Delta R(n)}=\left(\frac{n-2}{n-1}\right)^{n-1} \frac{S_{n}(n)}{I_{G}(n)}, \quad n \geq 2 .
$$

It is observed that the metric $\Delta r(n)$ in (19) corresponds to a weighted SGINR. For a specific case of $n=1$, it is obvious that selecting the user associated with the maximum SNR is optimal in terms of the sum rate. Hence, we define $\Delta r(1) \equiv \operatorname{det}\left(\rho_{i}^{(k)}\left(\mathbf{H}_{i, i}^{(k)}\right)\left(\mathbf{H}_{i, i}^{(k)}\right)^{H}\right)$. Note that $\Delta r(n)$, which is the proposed criterion for user selection, depends on the transmit beamforming vector for $n \geq 2$.

\subsection{User selection algorithm}

In this section, we propose a user selection algorithm with the objective of sum rate maximization. In the previous subsection, we have defined $\Delta r(n)$ as a criterion for user selection. In order to maximize the sum rate, BSs select users by using $\Delta r(n)$. If $\Delta r(n)$ is larger than 1 , which means that sum rate is increased by selecting the $n$th user, and the $n$th user is selected and added to $\mathcal{S}^{*}$. Otherwise, the $n$th user are not selected and the user selection procedure is terminated. Specifically, the proposed algorithm is described as the following three steps:

Step 1. Initialize as $\mathcal{S}^{*}(0)=\phi$ and $n=1$.

Step 2. Compute $\Delta r_{i}^{(k)}(n)$, and find $\left(i_{n}, k_{n}\right)$ such that

$$
\left(i_{n}, k_{n}\right)=\underset{(i, k) \in\left(U_{1} \cdots \cup U_{L}\right)-\mathcal{S}^{*}(n-1)}{\arg \max } \Delta r_{i}^{(k)}(n),
$$

where $\Delta r_{i}^{(k)}(n)$ denotes the $\Delta r(n)$ corresponding to the $k$ th MS in the $i$ th cell. where $\Delta r_{i}^{(k)}(n)$ denotes the $\Delta r(n)$ corresponding to the $k$ th MS in the $i$ th cell. Step 3. If $\Delta r_{i_{n}}^{\left(k_{n}\right)}(n)>1$, then set $\mathcal{S}^{*}(n)=\mathcal{S}^{*}(n-1)$ $\cup\left\{i_{n}, k_{n}\right\}, n=n+1$ and go back to step 2 ; otherwise, terminate the algorithm.

In step 1 , the set of selected users $\mathcal{S}^{*}(0)$ is initialized as an empty set. In step 2 , a user associated with the maximum $\Delta r(n)$ is chosen from among the users not in $\mathcal{S}^{*}(n-1)$. In step 3, if the value of $\Delta r(n)$ is greater than 1 , the corresponding user index is added to $\mathcal{S}^{*}(n)$ and the algorithm is repeated from step 2. Otherwise, the algorithm terminates and the final set of selected user is determined as $\mathcal{S}^{*}(n-1)$.

It should be noted that the proposed algorithm requires much lower computational complexity than the exhaustive search. Since the computational complexity of a user selection scheme mainly comes from computation of the precoding matrices, we measure the computational complexity in terms of the required number of computing precoding matrices. The proposed scheme requires at most $L\left(N_{\mathrm{t}} / N_{\mathrm{r}}\right)$ iterations and one user is selected at each iteration. Correspondingly, the complexity of the proposed scheme can be found to be $\sum_{i=1}^{N_{\mathrm{t}} / N_{\mathrm{r}}}(L K-i+1)$. The exhaustive search needs to compute precoding matrices for all possible sets of users, and the max-user exhaustive search also needs to compute precoding matrices for all possible sets of $\left(N_{\mathrm{t}} / N_{\mathrm{r}}\right)$ users. Based on these computations, the overall computational complexity of each scheme is tabulated in Table 1. It can be seen that the complexity of the proposed scheme is much lower than that of the exhaustive search and max-user exhaustive search. When $L=3$, $K=10, N_{\mathrm{t}}=4$, and $N_{\mathrm{r}}=2$, for instance, the overall computational complexity of the proposed scheme is 165 , whereas that of the exhaustive search and max-user exhaustive search is $4,397,880$ and $3,562,650$, respectively.

Table 1 Comparison of computational complexity

\begin{tabular}{lcc}
\hline & Proposed scheme & Exhaustive search \\
\hline Complexity $^{\mathbf{a}}$ & $\sum_{i=1}^{L\left(N_{\mathrm{t}} / N_{\mathrm{r}}\right)}(L K-i+1)$ & $\sum_{i=1}^{L\left(N_{\mathrm{t}} / N_{\mathrm{r}}\right)}\left(i \times L K C_{i}\right)$ \\
\hline
\end{tabular}

a The complexity is measured in terms of the required number of computing precoding matrices. 


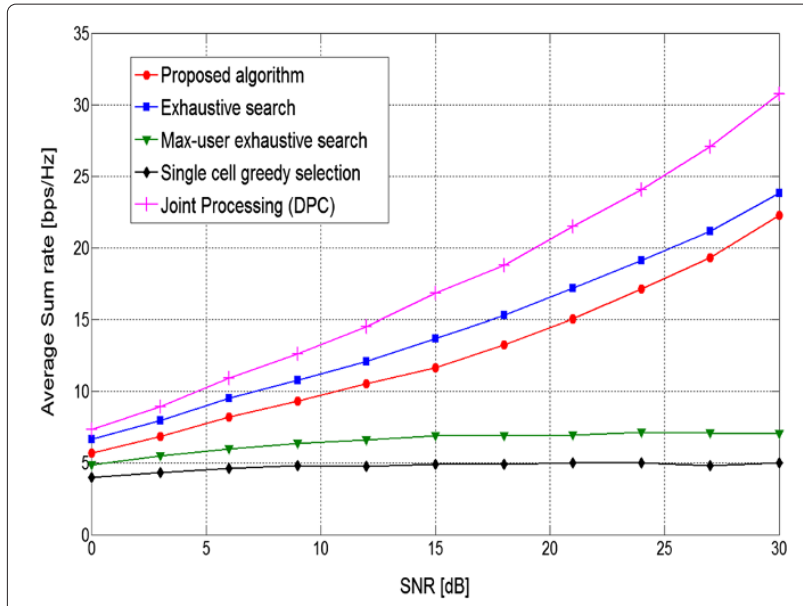

Figure 3 Average sum rate vs. SNR under the scenario 1, when $K=10, N_{\mathrm{t}}=4$ and $N_{\mathrm{r}}=2$.

\section{Numerical results}

In this section, we evaluate the performance of the proposed user selection scheme. We consider a cluster composed of three cells $(L=3)$, each of which corresponds to a sector of sectored cells, as depicted in Figure 1. The average channel gain between the $\mathrm{BS}$ in the $i$ th cell and the $k$ th MS in the cell is defined as $E\left[\left\|\mathbf{H}_{i, i}^{(k)}\right\|^{2}\right]=\rho_{0}\left(d_{i, k} / d_{\mathrm{r}}\right)^{-\alpha}$, where $d_{i, k}$ denotes the distance between a BS in the $i$ th cell and $k$ th MS, and $\rho_{0}$ denotes the SNR at a distance $d_{\mathrm{r}}$ from the base station. The reference distance $d_{\mathrm{r}}$ can be regarded as the cell radius. The values of $d_{\mathrm{r}}, \rho_{0}$, and the pathloss exponent $\alpha$ are set to $500 \mathrm{~m}, 8 \mathrm{~dB}$, and 3.7, respectively. The performance of the proposed scheme is compared with that of the exhaustive search, the maxuser exhaustive search, and the single-cell greedy selection in [11]. In addition, a joint processing scheme based on

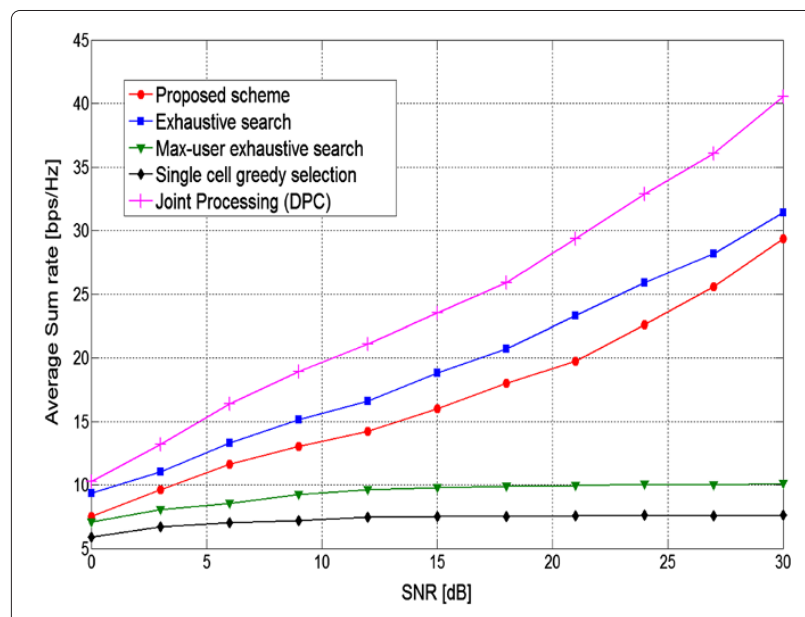

Figure 4 Average sum rate vs. SNR under the scenario 2 , when $K=10, N_{\mathrm{t}}=4$ and $N_{\mathrm{r}}=2$.

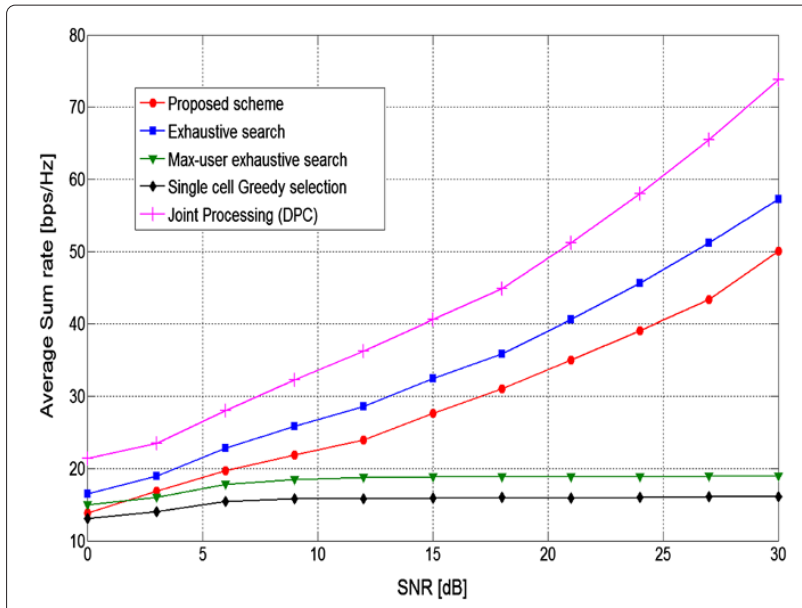

Figure 5 Average sum rate vs. SNR under the scenario 3, when $K=10, N_{\mathrm{t}}=4$ and $N_{\mathrm{r}}=2$.

the dirty paper coding (DPC) is used to provide an upper bound as in $[12,13]$. However, it should be noted that the joint processing requires sharing of data as well as the CSI among cells in each cluster, while the other schemes require sharing of only the CSI. In the exhaustive search, all the BSs in the cluster selects optimal user set that maximizes sum rate. The max-user exhaustive search is a modified version of the optimal exhaustive search, in which each BS always selects a set of $N_{\mathrm{t}} / N_{\mathrm{r}}$ users that maximizes the sum rate differently from the exhaustive search. The max-user exhaustive search can be considered as a form of coordinated greedy selection. In the singlecell greedy selection, each BS independently selects users associated with the maximum SINR without considering the intercell interference.

We consider three scenarios of user distribution to evaluate the performance of the coordinated user selection

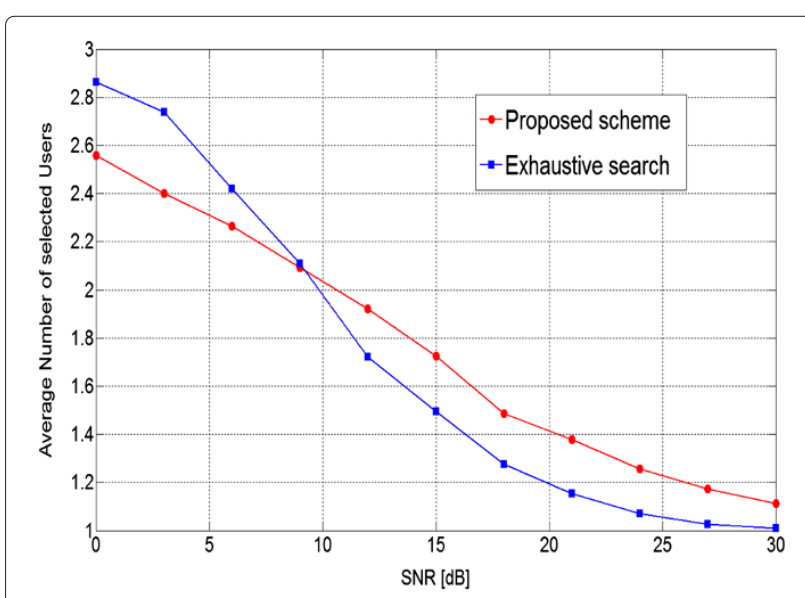

Figure 6 Average number of selected users vs. SNR under the scenario 1 , when $K=10, N_{\mathrm{t}}=4$ and $N_{\mathrm{r}}=2$. 


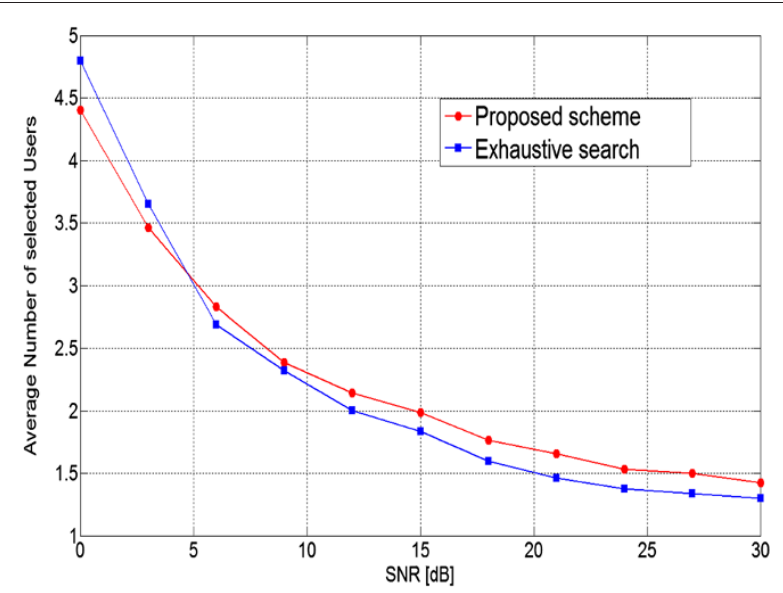

Figure 7 Average number of selected users vs. SNR under the scenario 2, when $K=10, N_{\mathrm{t}}=4$ and $N_{\mathrm{r}}=2$.

schemes. Scenario 1 corresponds to the case in which all users are located in cell edge areas in between $0.9 R$ and $R$, scenario 2 corresponds to the case where all the users are located in between $0.5 R$ and $R$, where $R$ denotes the cell radius, and scenario 3 corresponds to the case where all the users are located uniformly over the entire cell. The reason why we consider scenarios 1 and 2 is that users around cell center can be served separately by each cell without coordination, since the impact of the intercell interference will be limited $[10,14]$. Figures 3 , 4, 5 depict the achievable sum rate and Figures 6, 7, 8 illustrate the average number of selected users vs. SNR under these three scenarios, respectively, when $K=10$, $N_{\mathrm{t}}=4$, and $N_{\mathrm{r}}=2$. From Figures 3, 4, 5, the proposed scheme is shown to significantly outperform the singlecell greedy selection and the max-user exhaustive search. Moreover, the performance degradation as compared to

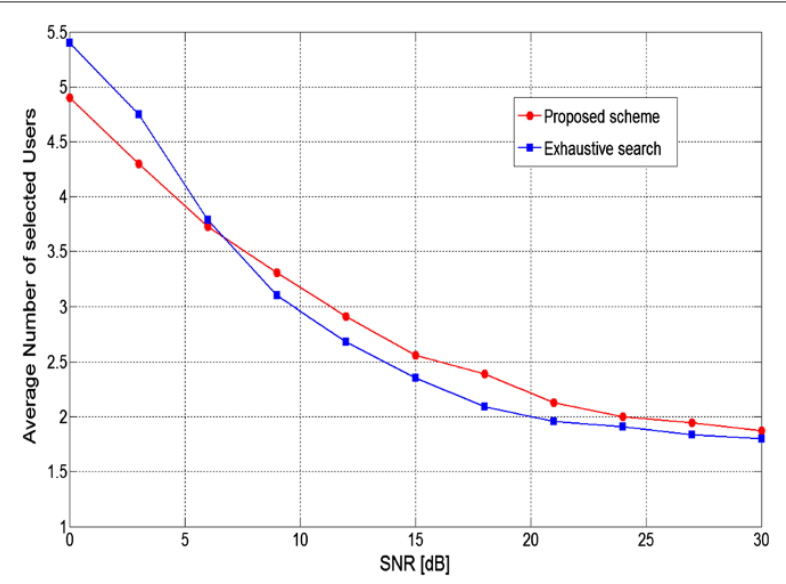

Figure 8 Average number of selected users vs. SNR under the scenario 3, when $K=10, N_{\mathrm{t}}=4$ and $N_{\mathrm{r}}=2$. the exhaustive search seems not that much considering the substantial reduction in the complexity. For instance, when $L=3, K=10, N_{\mathrm{t}}=4$, and $N_{\mathrm{r}}=2$, the proposed scheme achieves about $94 \%$ of the sum rate of the exhaustive search, while the reduction in computational complexity amounts to about 26,600-fold. As expected, the performance of the joint processing is better than the other schemes at the cost of the backhaul overhead for data sharing among cells. From Figures 6, 7,8 , it can be observed that not all the degrees of freedoms at the BS are used to transmit as many streams as possible. This suggests that using all the degrees of freedoms is not always optimal for sum rate. Some degrees of freedoms need to be used for interference mitigation, in such a way that the average sum rate is maximized. As an extreme case, when the SNR is sufficiently high, the proposed solution is shown to approach the single-user scheme, as discussed in [2]. The results in Figure 5 also verify that the performance gain of the proposed scheme is substantial even when users are uniformly distributed throughout the cell.

\section{Conclusions}

In this article, we have proposed a successive user selection scheme for the downlink of MIMO cellular systems in a multicell environment. The proposed scheme works jointly with SGINR beamforming and attempts to maximize the sum rate over all of the users in a cluster of cells. As compared to the optimal exhaustive search, the proposed scheme is much less complex due to the derivation of a simple incremental metric for the sum rate. Numerical results confirm that the proposed user selection scheme achieves a sum rate close to that of the exhaustive search. In a particular case, the proposed scheme has been shown to achieve $94 \%$ of the sum rate of the exhaustive search, while reduction in computational complexity amounts to about 26,600 -fold.

\section{Endnote}

${ }^{a}$ It should be noted that the approximation is used just to simplify the incremental metric for user selection. The high SINR assumption should not restrict the derived metric to being used only at high SINR. In Section 4, we will consider a situation in which all the MSs are distributed over the cell edge area where the SINR is generally low.

\section{Competing interests}

The authors declare that they have no competing interests.

\section{Acknowledgements}

This work was supported by the National Research Foundation of Korea (NRF) grant funded by the Korea government (MEST) (no. 20110020262).

\section{Author details}

${ }^{1}$ School of Electrical and Computer Engineering and INMC, Seoul National University, 151-742 Seoul, South Korea. ${ }^{2}$ School of Electronic Engineering, Soongsil University, Seoul 156-743, South Korea. 


\section{References}

1. S Catreux, PF Driessen, LJ Greenstein, Simulation results for an interference-limited multiple-input multiple-output cellular system. IEEE Commun. Lett. 4(11), 334-336 (2000)

2. S Ye, RS Blum, Optimized signaling for MIMO interference systems with feedback. IEEE Trans. Signal Process. 51(11), 2839-2848 (2003)

3. M Sadek, A Tarighat, AH Sayed, A leakage-based precoding scheme for downlink multi-user MIMO channels. IEEE Trans. Wireless Commun. 6(5), 1711-1721 (2007)

4. RS Blum, MIMO capacity with interference. IEEE J. Select. Areas Commun. 21(5), 793-801 (2003)

5. BO Lee, HW Je, OS Shin, KB Lee, A novel uplink MIMO transmission scheme in a multicell environment. IEEE Trans. Wireless Commun. 8(10), 4981-4987 (2009)

6. KN Lau, Analytical framework for multiuser uplink MIMO spacetime scheduling design with convex utility functions. IEEE Trans. Wireless Commun. 3(5), 1832-1843 (2004)

7. Y Hara, L Brunel, K Oshima, in Proceedings of the IEEE International Symposium on Personal, Indoor \& Mobile Radio Communications. Uplink spatial scheduling with adaptive transmit beamforming in multiuser MIMO systems (Helsinki Finland, 11-14 September 2006)

8. S Serbetli, A Yener, in Proceedings of the Asilomar Conference on Signals, Systems \& Computers. Beamforming and scheduling strategies for time slotted multiuser MIMO systems (Pacific Grove, CA, USA, p. 7-10 November 2004

9. 3GPP, Leakage-based precoding for CoMP in LTE-A. 3GPP R1-090028 (2009). http://www.3gpp.org/ftp/tsg_ran/wg1_rl1/TSGR1_55b/Docs/R1090028.zip

10. 3GPP, Coordinated multi-point operation for LTE physical layer aspects (release 11). 3GPP TR 36.819, V11.1.0 (2011). http://www.3gpp.org/ftp/ Specs/archive/36_series/36.819/36819-110.zip

11. G Dimic, ND Sidiropoulos, On downlink beamforming with greedy user selection: performance analysis and a simple new algorithm. IEEE Trans. Signal Process. 53(10), 3857-3868 (2005)

12. P Patcharamaneepakorn, A Doufexi, S Armour, in Proceedings of the IEEE Vehicular Technology Conference. Reduced complexity joint user and receive antenna selection algorithms for SLNR-based precoding in MU-MIMO systems Yokohama, 6-9 May 2012)

13. TYoo, A Goldsmith, On the optimality of multiantenna broadcast scheduling using zero-forcing beamforming. IEEE J. Select. Areas Commun. 24(3), 528-541 (2006)

14. 3GPP, Further consideration on enhanced SRS for CoMP/non-CoMP user group. 3GPP R1-095087 (2009). http://www.3gpp.org/ftp/tsg_ran/ wg1_rl1/TSGR1_59/Docs/R1-095087.zip

doi:10.1186/1687-1499-2013-154

Cite this article as: Kim et al: A low-complexity user selection scheme in a multicell MIMO environment. EURASIP Journal on Wireless Communications and Networking 2013 2013:154.

\section{Submit your manuscript to a SpringerOpen ${ }^{\mathcal{O}}$ journal and benefit from:}

- Convenient online submission

- Rigorous peer review

- Immediate publication on acceptance

- Open access: articles freely available online

- High visibility within the field

- Retaining the copyright to your article

Submit your next manuscript at $\boldsymbol{\wedge}$ springeropen.com 